\title{
Real Power Loss Minimization and Voltage Stability Limit Enhancement by Using Shuffled Frog Leap Algorithm
}

\author{
P. B. Chennaiah ${ }^{1}$, N. Krishna Kishore ${ }^{2}$, Dr. M. Suryakalavathi ${ }^{3}$ \\ ${ }^{1}$ Assistant professor, Department of Electrical \& Electronics Engineering, Annamacharya Institute of \\ Technology \& science. \\ ${ }^{2} P G$ student, Department of Electrical \& Electronics Engineering, Annamacharya Institute of Technology \& \\ science. \\ ${ }^{3}$ Professor, Department of Electrical \& Electronics Engineering, JNTUH,
}

\begin{abstract}
This paper deals with the Optimal location and control of a unified power flow controller (UPFC) along with transformer taps are tuned to simultaneously optimize the voltage stability limit (VSL) and real power losses of a mesh power system network. This problem is dealt as a nonlinear equality and inequality constrained optimization problem with an objective function incorporating both the real power loss and VSL. An evolutionary algorithm known as shuffled frog leap is applied for solving the UPFC location, its injected series voltage, and also the tap positions of transformers as the variables. The then obtained results of SFC algorithm are compared with the results of Bacteria Foraging-Based Algorithm using IEEE 14 bus.
\end{abstract}

Index Terms: Shuffled frog leap, continuation power flow, linearprogramming, optimal power flow (OPF).

\section{Introduction:}

The OPF methods are conventional and intelligent and solved by varieties of methods such as successive linear programming, the Newton-based nonlinear programming method, and with varieties of recently proposed interior point methods. The Optimal Power Flow solution is used to optimize a selected objective function such as fuel cost with optimal adjustment of the power system control variables, at the same time satisfying various equity and inequality constraints. The drawback of the OPF is solved from different perspectives, like analyzing the effects of load on voltage stability/power flow solvability, generation rescheduling for cost minimization of power generation, controls such as taps, shunts, and other modern VAR sources adjustments to minimize real power losses in the system.

The advent of Flexible ac transmission systems (FACTS) system made the possibility for optimizing the power flow without the restoration of generation rescheduling or changes to topology. Unified power flow controller (UPFC) is the advanced in the controllers family and can provide the OPF with significant flexibility by injecting compensation in series and shunt in controlled manner.

The UPFC can provide simultaneous control of all basic power system parameters (transmission voltage, impedance and phase angle). The controller can fulfil functions of reactive shunt compensation, series compensation and phase shifting meeting multiple control objectives. From a functional perspective, the objectives are met by applying a boosting transformer injected voltage and a exciting transformer reactive current. The injected voltage is inserted by a series transformer. The continuation power flow (CPF) method is robust; however has some weakness in large electric power system considering generators reactive power limits and gives information regarding how much percentage overloading the system can withstand before a possible voltage collapse. The CPF problem is incorporated into an OPF problem so that both the issues can be addressed simultaneously. In this paper, the voltage stability limit is defined as the maximum percentage overloading $\left(\lambda_{\max }\right)$ the system can withstand and incorporated along with the objective of real power loss minimization.

The classical techniques of OPF solution has the disadvantage that they are sensitive to starting points and leading to non-monotonic solution. To eliminate this problem evolutionary techniques have been applied in solving the OPF problem [10], [11] like particle swarm optimization (PSO) to the problem of OPF.

In this paper a new evolutionary algorithm known as Shuffled Frog Leap algorithm (SFLA) is used to solve the combined problem of CPF-OPF for real power loss minimization and VSL maximization of the system. The algorithm has been inspired from memetic evolution of a group of frogs when seeking for food. In this method, a solution to a given problem is presented in the form of a string, called "frog" which has been considered as a control vector in this paper. The main objective is transformer taps optimization, location of UPFC and its series injected voltage for the single objective of real power loss minimization and then for the multiple objectives of loss minimization and VSL maximization.

Finally, a cost analysis for installation of UPFC is carried out to establish the investment in putting a UPFC for the cause. Test results indicate that SFLA method can obtain better results than the simple heuristic search method on the 14-bus radial distribution systems. 


\section{Problem Formulation for Optimal Power Flow (OPF)}

\section{Statement Of The Problem:}

The UPSC is a unique device that can provide simultaneous control of all basic power system parameters. It can fulfil the functions of reactive shunt compensation, series compensation and phase shifting meeting multiple control objectives. UPSC consists of series and shunt connected converters as in Fig.1.

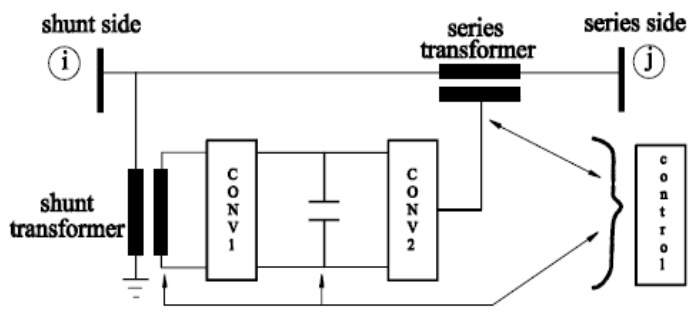

Fig.1. Basic arrangement of UPFC.

The UPSC can control the line's real power and reactive power and bus voltage where it is connected, by proper injection of voltage magnitude in series and shunt respectively. In this paper the UPSC with injection model [6] is connected at suitable location in the system. The injection model of upsc is shown in Fig. 2.

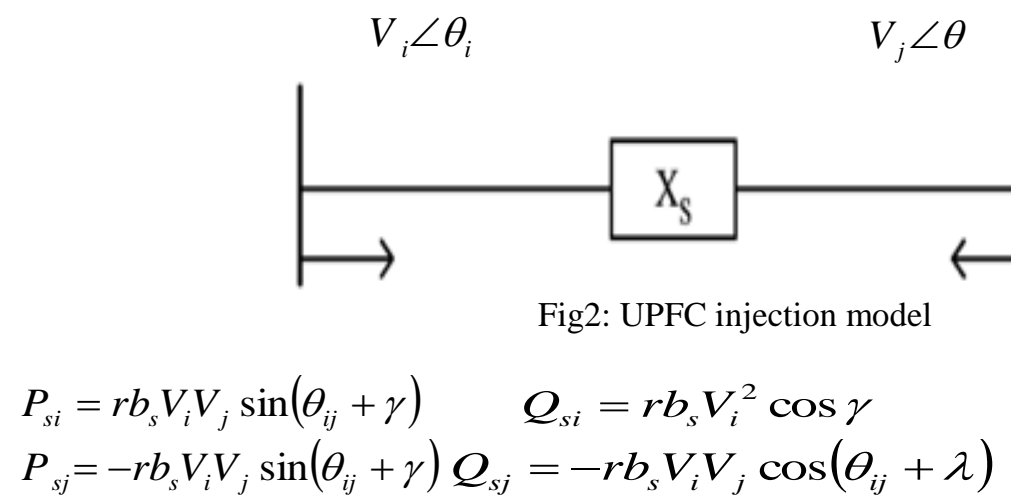

The OPF problem solution aims to optimize a selected objective function such as fuel cost via optimal adjustment of the power system control variables, while at the same time satisfying various equality and in equality constraints. The equality constraints are the power flow equations, while the in equality constraints are the limits on control variables and the operating limits of power system dependent variables. The problem control variables include the generator real powers, the generator bus voltages, the transformer tap settings, and the reactive power of switchable VAR sources, while the problem dependent variables include the load bus voltages, the generator reactive power $\mathrm{s}$, and the line flows.

To solve the set of nonlinear equations describing the optimal solution of power system it is expressed as

$$
\begin{array}{ll}
\text { Minimize } & F(x, u) \\
\text { Subject to } & g(x, u)=0 \\
& h(x, u) \leq 0 .
\end{array}
$$

The real power loss of the mesh connected multi machine test system is denoted by objective function $F(x, u)$, the set of nonlinear equality constraints to represent power flow by $g(x, u)$, and $h(x, u)$ is a set of nonlinear inequality constraints (i.e., bus voltages, transformer/line MVA limits, etc.). " $x$ " is a vector of dependent variables and " $u$ " is a vector of control variables. Here, the transformer tap values are the control variables, and both the magnitude and phase angle of UPFC series injected voltage $\left(V_{s c}\right)$.

\section{Voltage Stability Limit with $O P F$ :}

With the maximization of VSL the real power loss minimization can be augmented. The VSL can be calculated through CPF. The CPF introduces a load parameter defined as the percentage increase of generation and load from its base value. The resulting load and generation equation in terms of the load parameter is as follows: 


$$
\begin{aligned}
& P_{L i}=P_{L i 0}(1+\lambda) \\
& Q_{L i}=Q_{L i}(1+\lambda) \\
& P_{L i}=P_{G i 0}(1+\lambda)
\end{aligned}
$$

The load parameter can be increased until the "notch point" (the point at which the system reaches to instability) of the PV-curve. The maximum value of the load parameter is termed as VSL. The objective is to

$$
\text { Optimize } \quad F\left(x, u, \lambda_{\text {macx }}\right)
$$

$$
\text { Subject to } \quad g(x, u)=0
$$

$$
h(x, u) \leq 0
$$

The function to be optimized now can be represented as

$$
F\left(x, u, \lambda_{\max }\right)=G(x, u)+V\left(\lambda_{\text {max }}\right)
$$

where

$$
\begin{gathered}
\mathrm{G}(\mathrm{x}, \mathrm{u})=\text { Real Power Loss } \\
V\left(\lambda_{\max }\right)=1 / \lambda_{\max }
\end{gathered}
$$

The solution of CPF is carried out with the help of a suitably chosen continuation parameter. With the increase of " $\lambda$ " a new solution point is predicted first and then corrected in usual predictor and corrector steps. Since the objective is to maximize the VSL, so its reciprocal is added to the original cost function of real power loss so that the overall cost can be minimized.

\section{Shuffled Frog Leap Algorithm:}

The SFLA-based approach for solving the optimal placement and sizing of distributed generation problem to minimize the loss and improve the voltage profile takes the following steps: In SFLA, each possible solution $\mathrm{Xi}=(\mathrm{xi} 1, \mathrm{xi} 2, \ldots, \mathrm{xiS})$ that in this paper $\mathrm{Xi}=11,12, \ldots, \mathrm{lbus}, \mathrm{x} 1, \mathrm{x} 2, \ldots, \mathrm{xpower}$ limit Where, 1 is the number of DG location candidates and $\mathrm{x}$ is the number of capacity types of DGs are is considered as a frog. The steps of the algorithm are as follows:

Step 1: Create an initial population of $\mathrm{P}$ frogs generated randomly. SFLA Population $=[\mathrm{X} 1, \mathrm{X} 2, \ldots, \mathrm{Xp}] \mathrm{p} \times \mathrm{n}$ Where, $\mathrm{P}=\mathrm{m} \times \mathrm{n}, \mathrm{N}$ is the number of $\mathrm{DG}, \mathrm{m}$ is the number of memplexes and $\mathrm{n}$ is the number of frogs in memplex.

Step 2: Sort the population increasingly and divide the frogs into $\mathrm{m}$ memplexes each holding $\mathrm{n}$ frogs such that $\mathrm{P}=\mathrm{m} \times \mathrm{n}$. The division is done with the first frog going to the first memplex, second one going to the second memplex, the mth frog to the mth memplex and the m+lth frog back to the first memplex. Fig. 3 illustrates this memeplex partitioning process.

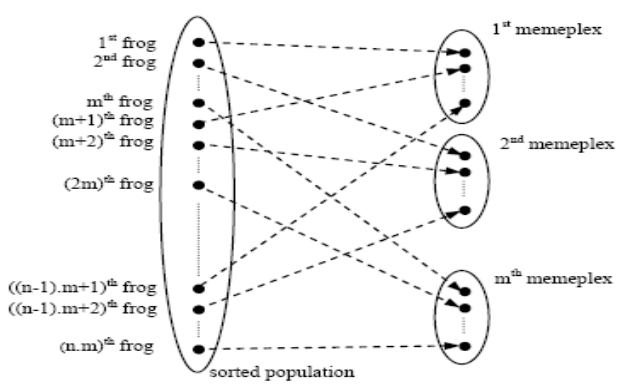

Fig.3. Memeplex partitioning process

Step 3: Within each constructed memeplex, the frogs are infected by other frogs' ideas; hence they experience a me-metic evolution. Memetic evolution improves the quality of the meme of an individual and enhances the individual frog's performance towards a goal. Below are details of memetic evolutions for each memeplex:

Step 3-1: Set $\mathrm{m} 1=0$ where $\mathrm{m} 1$ counts the number of me-meplexes and will be compared with the total number of memeplexes $\mathrm{m}$. Set $\mathrm{y} 1=0$ where $\mathrm{y} 1$ counts the number of evolutionary steps and will be compared with the maximum number of steps (ymax), to be completed within each meme-plex. Step 
3-2: Set $\mathrm{m} 1=\mathrm{m} 1+1$ Step 3-3: Set $\mathrm{y} 1=\mathrm{y} 1+1$ Step 3-4: For each memplex, the frogs with the best fitness and worst fitness are identified as Xw and $\mathrm{Xb}$ respectively. Also the frog with the global best fitness Xg is identified, and then the position of the worst frog Xw for the memplex is adjusted such as (10) and (11). Fig.5. demonstrates the original frog leaping rule.

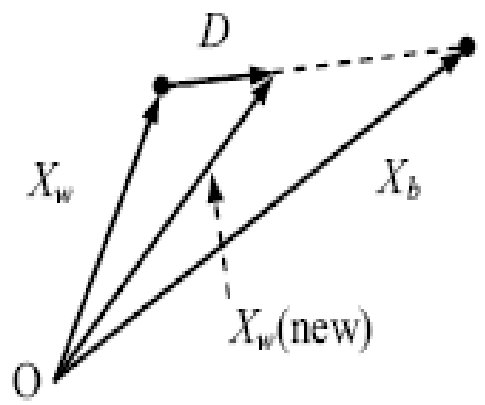

Figure 4.The original frog leaping rule

If the evolutions produce a better frog (solution), it re-places the older frog, otherwise $\mathrm{Xb}$ is replaced by $\mathrm{Xg}$ in (10) and the process is repeated. If no improvement becomes possible in this case a random frog is generated which re-places the old frog. Step 3-5: If $\mathrm{m} 1<\mathrm{m}$, return to step3-2. If y1<ymax, return to step 3-3, otherwise go to step 2. Step 4: Check the convergence. If the convergence criteria are satisfied stop, otherwise consider the new population as the initial population and return to the step2. The best solution found in the search process is considered as the output results of the algorithm. The flowchart of the SFLA is illustrated in Fig. 3.

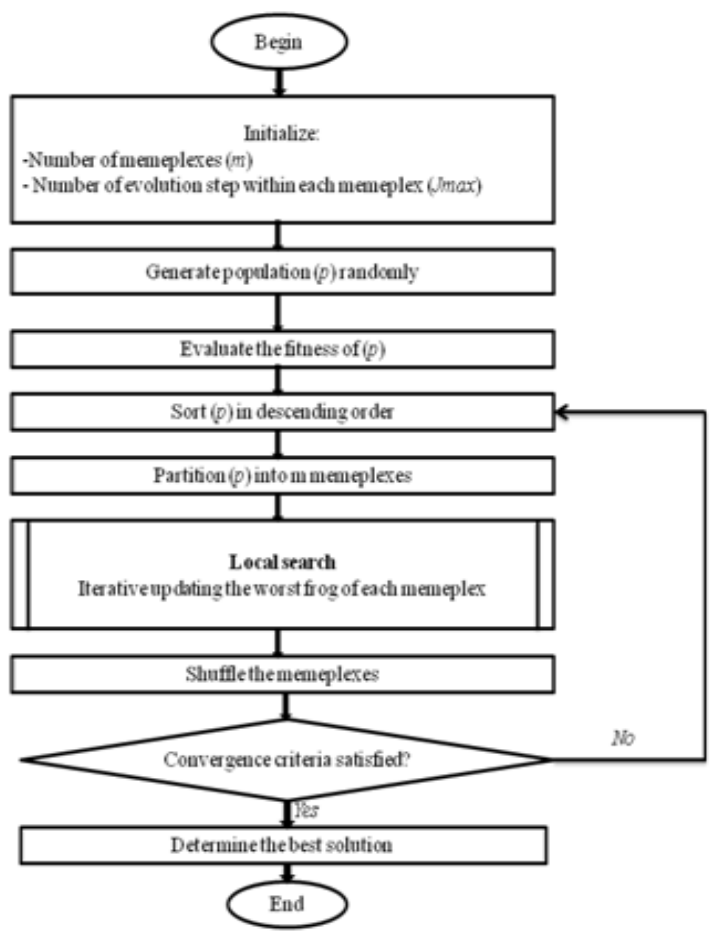

Fig.5. Flowchart of the SFLA

\section{Results And Discussion:}

The objective function for the multi-objective optimization can be formulated as $F=p f_{1}+p f_{2}+p f_{3}+o f+V\left(\lambda_{\max }\right)$

Where $p f_{1}, p f_{2}$, and $p f_{3}$ are the penalty factors and of is the real power loss. Vmax and Vmin are the maximum and minimum limits of bus voltages [14] for all the buses. Similarly, trans $_{\max }$ and line $_{\max }$ are, respectively, the maximum MVA limits of the transformers and lines in the system. 
With an objective to optimize both real power loss and VSL, the cost function is modified by reciprocal of VSL to the real power loss. The optimization is carried out by SFLA. The transformer tap values along with the corresponding optimized loss and VSL are given in Table 1. It is seen that the VSL value has improved, although the real power loss has increased marginally. However, the sum of real power loss and the reciprocal of VSL has reduced, when the multi-objective function is considered.

Table 1: Simultaneous Optimized Values of UPFC and Transformer Taps for BFAM

\begin{tabular}{|c|c|c|c|c|c|}
\hline \multicolumn{7}{|c|}{ Optimized Taps And Parameters For Real Power Losses And } \\
Voltage Stability Limit For BFAM
\end{tabular}

Table 2: Simultaneous Optimized Values of UPFC and Transformer Taps for SFLA

\begin{tabular}{|c|c|c|c|c|c|c|c|}
\hline \multicolumn{8}{|c|}{$\begin{array}{c}\text { Optimized Taps And Parameters For Real Power Losses And } \\
\text { Voltage Stability Limit For SFLA }\end{array}$} \\
\hline S.No & $\begin{array}{l}\text { Line } \\
\text { No }\end{array}$ & $\begin{array}{c}\text { Transf } \\
\text { ormer } \\
\text { Taps }\end{array}$ & & $\begin{array}{l}\text { ajected } \\
\text { Joltage }\end{array}$ & & $\begin{array}{c}\text { Real } \\
\text { Power } \\
\text { Losses }\end{array}$ & $\begin{array}{c}\text { Voltage } \\
\text { Stability } \\
\text { Limit }\end{array}$ \\
\hline 1 & $2-30$ & 1.00 & \multirow{12}{*}{ 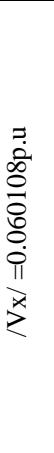 } & \multirow{12}{*}{\multicolumn{2}{|c|}{ 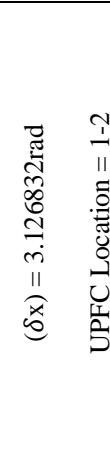 }} & \multirow{12}{*}{ 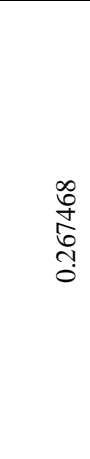 } & \multirow{12}{*}{ 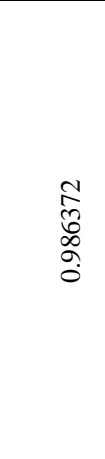 } \\
\hline 2 & $10-32$ & 1.13 & & & & & \\
\hline 3 & $12-11$ & 1.02 & & & & & \\
\hline 4 & $12-13$ & 1.11 & & & & & \\
\hline 5 & $19-33$ & 1.14 & & & & & \\
\hline 6 & $19-20$ & 1.00 & & & & & \\
\hline 7 & $20-34$ & 1.08 & & & & & \\
\hline 8 & $22-35$ & 1.07 & & & & & \\
\hline 9 & $23-36$ & 0.95 & & & & & \\
\hline 10 & $25-37$ & 1.06 & & & & & \\
\hline 11 & $29-38$ & 1.11 & & & & & \\
\hline 12 & $6-31$ & 1.15 & & & & & \\
\hline
\end{tabular}

With the aim of till reducing the objective function the UPFC variables are evaluated by the optimized transformer tap positions obtained in previous step. The UPFC is connected at the left-hand-side bus as per line notation given in [15]. In the case of SFLA, the line at which UPFC should be connected is decided randomly out of 32 lines selected in the initial stage. So with SFLA, the line number in which UPFC is to be connected also becomes a control variable along with the others. The UPFC is connected in all the 32 lines, considering one at a time. The best location and the UPFC injection voltage in each succession of linearization are retained. The overall cost function can still be reduced by simultaneous optimization of UPFC location and its variables along with transformer taps. The numbers of variables now become 15, i.e., 12 transformer tap positions, and three UPFC variables. With SFLA, this becomes 14, as the location of UPFC cannot be taken. The optimization algorithm remains the same. It is seen that, the VSL has improved considerably, though at the cost of deteriorated loss reduction. The P-V curves for the three optimized schemes are shown in Fig.7. 


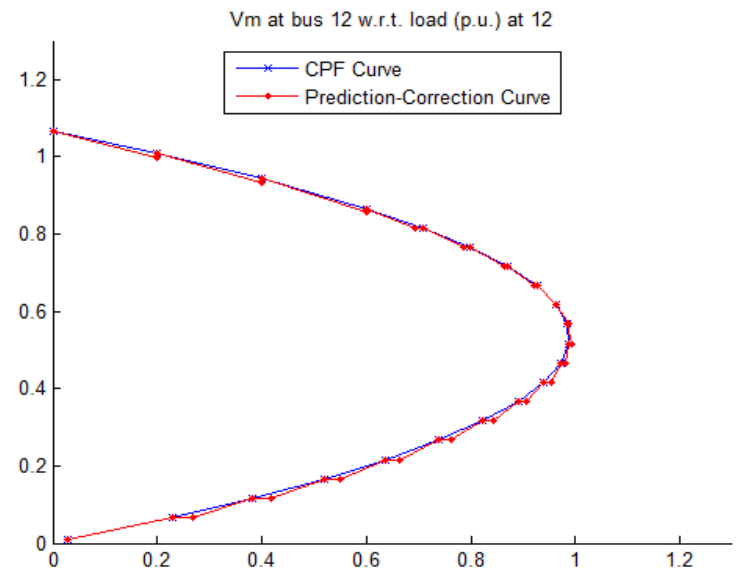

Fig.6. P-V curves

To show the efficiency of the proposed method the voltage profile obtained from the SFLA is compared with the voltage profile obtained from BFAM. The voltage profile of all the buses at nominal load is shown in Fig.7 and Fig.8.

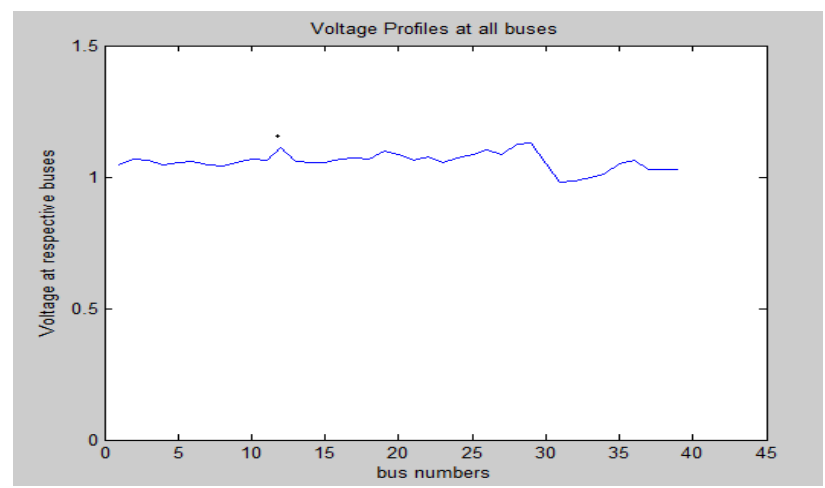

Fig.7 Voltage profile at all buses with BFAM

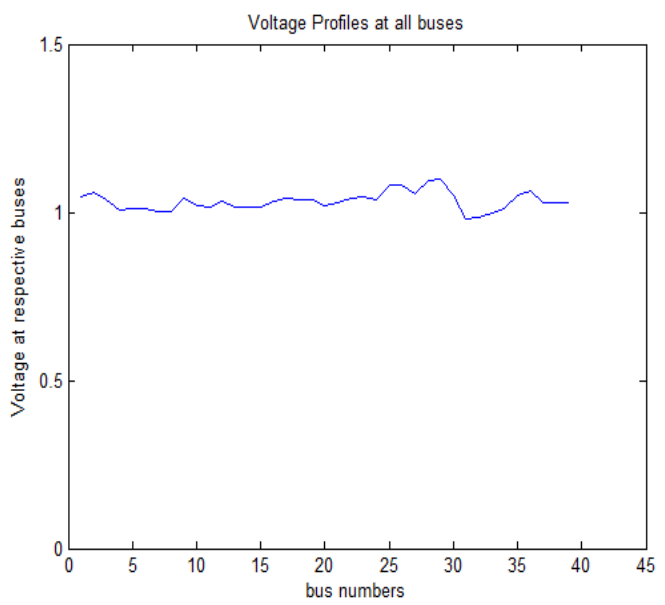

Fig. 7 Voltage profile at all buses with SFLA

It is seen that all the bus voltages remain within the limits, and the generator buses maintain their specified voltages when the optimized variables are used.

\section{Conclusion:}

This paper presented an Shuffled Frog Leap Algorithm is used for allocating, transformer taps, and UPFC with a view to minimize the real power loss and improve VSL of a system simultaneously. The performance of the Shuffled Frog Leap algorithm for solving multi-objective that is real power loss 
minimization and Maximization of Voltage stability limit is demonstrated using IEEE-14 bus test system. The results shows that the SFLA algorithm which is used for Allocating transformer taps, and control of UPFC with a view to simultaneously minimize the real power loss and maximize the Voltage stability (VSL) of the system. The results of the multi-objective solution show that the SFLAM technique has provided the better solution as compared to the BFAM.

\section{References}

[1] P. Ristanovic, "Successive linear programming based OPF solution," Optimal Power Flow: Solution Techniques, Requirements and Challenges,IEEE Power Eng. Soc., pp. 1-9, 1996.

[2] D. Sun et al., "Optimal power flow by newton approach," IEEE Trans. Power App. Syst., vol. PAS-103, no. 10, pp. 2864-2875, Oct. 1984

[3] S. Granville, "Optimal power dispatch through interior pont methods," IEEE Trans. Power Syst., vol. 9, no. 4, pp. 1780-1787, Nov. 1994.

[4] G. Torres and V. Quintana, "An interior point method for non-linear optimal power flow using voltage rectangular coordinates," IEEE Trans.Power Syst., vol. 13, no. 4, pp. 1211-1218, Nov. 1998.

[5] J. L. Martinez Ramos, A. G. Exposito, and V. Quintana, "Transmission loss reduction by interior point methods: implementation issues and practical experience,” Proc. Inst. Elect. Eng., Gen., Transm., Distrib., vol. 152, no. 1, pp. 90-98, Jan. 2005.

[6] M. Noroozian, L. Angquist, M. Ghandhari, and G. Anderson, "Use of UPFC for optimal power flow control," IEEE Trans. Power Del., vol. 12, no. 4, pp. 1629-1634, Oct. 1997.

[7] G. Glanzmann and G. Andersson, "Coordinated control of FACTS devices based on optimal power flow," in Proc. 37th Annu. North Amer.Power Symp., Ames, IA, Oct. 23-25, 2005.

[8] V. Ajjarapu and C. Christy, "The continuation power flow: a tool for steady state voltage stability analysis," IEEE Trans. Power Syst., vol. 7, no. 1, pp. 416-423, Feb. 1992.

[9] F. Milano, C. A. Canizares, and A. J. Conejo, "Sensitivity-based security- constrained OPF market clearing model," IEEE Trans. Power Syst., vol. 20, no. 4, pp. 2051-2060, Nov. 2005.

[10] J. Yuryevich and K. P. Wong, "Evolutionary programming based optimal power flow algorithm," IEEE Trans. Power Syst., vol. 14, no. 4, pp. $1245-1250$, Nov. 1999.

[11] A. A. A. Esmin, G. Torres, and A. C. Z. de Souza, "A hybrid particle swarm optimization applied to loss power minimization," IEEE Trans.Power Syst., vol. 20, no. 2, pp. 859-866, May 2005.

[12] M. Eusuff, K. Lansey, F. Pasha; "Shuffled frog-leaping algorithm: a memetic meta-heuristic for discrete optimiza-tion", Engineering Optimization, 2006, Vol. 38, No. 2, pp.129-154.

[13] Xuncai Zhang1,2, Xuemei Hu3, Guangzhao Cui2, Yanfeng Wang2, Ying Niu2," An Improved Shuffled Frog Leaping Algorithm with Cognitive Behavior", Proceedings of the 7th World Congress on Intelligent Control and Automation June 25 - 27, 2008, Chongqing, China.

[14] M. Saravan, S. M. Raja Slochanal, P. Venkatesh, and J. P. S. Abraham, "Application of PSO technique for optimal location of FACTS devices considering system loadability and cost of installation," in Proc. $7^{\text {th }}$ Int. Power Engineering Conf., 2005, Nov. 29Dec. 2, 2005. 